\title{
EFEITO DO VIGOR E TRATAMENTO DE SEMENTES DE SOJA COM AMINOÁCIDOS NO DESEMPENHO DAS SEMENTES PRODUZIDAS
}

\author{
Caio Sippel Dörr ${ }^{1}$, Tainan Lopes de Almeida², Vinicius Guilherme Kiesow Macedo³, Jonas Albandes \\ Gularte $^{4}$, Vinicius Diel5, Luís Eduardo Panozzo ${ }^{6}$
}

\begin{abstract}
${ }^{1}$ Engenheiro agrônomo, doutor em Ciência e Tecnologia de Sementes pela Universidade Federal de Pelotas. Email: caiodorrcsd@gmail.com. ${ }^{2}$ Engenheiro agrônomo, mestre em Ciência e Tecnologia de Sementes pela Universidade Federal de Pelotas ${ }^{3,4}$ Engenheiro agrônomo, doutorando em Ciência e Tecnologia de Sementes pela Universidade Federal de Pelotas, ${ }^{5}$ Engenheiro agrônomo, mestrando em Ciência e Tecnologia de Sementes pela Universidade Federal de Pelotas ${ }^{6}$ Engenheiro agrônomo Universidade Federal de Pelotas, doutorado em Fitotecnia Universidade Federal de Viçosa. Professor adjunto
\end{abstract} Faculdade de Agronomia Eliseu Maciel Universidade Federal de Pelotas.

RESUMO: A aplicação de aminoácidos em plantas tem como objetivo ativar o metabolismo vegetal, portanto, a sua aplicação em sementes de diferentes níveis de qualidade fisiológica pode promover o desempenho das plantas e resultar na produção de sementes de desempenho fisiológico superior. O objetivo do trabalho foi avaliar o desempenho fisiológico das sementes de soja produzidas por plantas oriundas de sementes de diferentes níveis de qualidade tratadas com doses de aminoácidos. O delineamento experimental foi 0 de blocos casualizados em esquema fatorial com quatro repetições, envolvendo a combinação de dois fatores. Fator A, lotes de sementes de três níveis de qualidade fisiológica (elevada, intermediária e reduzida), fator $\mathrm{B}$, cinco doses de produto comercial a base de aminoácidos utilizado em tratamento de sementes. As sementes foram tratadas com produto comercial (P.C.) a base de aminoácidos nas doses de 0, 200, 400,600, e $800 \mathrm{~mL}$ P.C. $100 \mathrm{~kg}^{-1}$ de sementes. Posteriormente, realizou-se semeadura em campo e na maturação as sementes foram colhidas e sua qualidade avaliada, mediante percentual de germinação, teste de frio, teste de envelhecimento acelerado e comprimento de parte aerea e raiz. Plantas de soja oriundas de sementes de elevada qualidade fisiológica produzem sementes de desempenho fisiológico superior. $O$ tratamento das sementes de soja utilizadas na semeadura com doses de aminoácidos não apresentou efeito significativo no desempenho fisiológico das sementes produzidas.

Palavras-chave: Glycine max, produção de sementes, fisiologia, qualidade fisiológica. 


\title{
EFFECT OF VIGOR AND TREATMENT OF SOYBEAN SEEDS WINH AMINO ACIDS ON THE PERFORMANCE OF SEEDS PRODUCED
}

\begin{abstract}
The application of amino acids in plants has as main objective the activation of plant metabolism, and its application in seeds of different levels of physiological quality can promote the performance of the plants in the field and result in the production of seeds of higher physiological performance. The objective of this work was to evaluate the physiological performance of soybean seeds produced by plants of different quality levels treated with doses of amino acids. The experimental design was a randomized complete block design with four replications, involving a combination of two factors. A factor, seed lots of three levels of physiological quality (high, intermediate and reduced), and factor $B$, five doses of commercial product based on amino acids used in seed treatment. Seeds of different levels of physiological quality were treated with commercial product (P.C.) at the doses of 0 , 200, 400, 600, and $800 \mathrm{~mL}$ P.C. $100 \mathrm{~kg}^{-1}$ of seeds. Seeds were harvested and their physiological quality evaluated by germination percentage, cold test, accelerated aging test and aerea and root length. Soybean plants from seeds of high physiological quality produce seeds of superior physiological performance. The treatment of soybean seeds used in sowing at doses of amino acids did not present a significant effect on the physiological performance of the seeds produced.
\end{abstract}

Keywords: Glycine max, seed production, physiology, physiological quality.

\section{INTRODUÇÃO}

A soja é uma cultura de forte expressão socioeconômica para o Brasil, pois vem desenvolvendo diferentes regiões do país, gerando grande demanda por mão de obra, e consequentemente o desenvolvimento da região. A região sul do Brasil representa $35,58 \%$ da produção nacional de grãos de soja. Devido ao desenvolvimento de novas tecnologias e as condições ambientais favoráveis para o bom desenvolvimento da cultura, todos os estados da região sul apresentaram safras recordes, onde o Rio Grande do Sul apresentou um incremento de produção de aproximadamente $15,51 \%$ em relação à safra anterior (2015/16), sendo que a área semeada e a produtividade gaúcha na cultura da soja apresentaram incrementos de $2,11 \%$ e 13,13\%, respectivamente (CONAB, 2017).

A utilização de sementes de elevada qualidade fisiológica tem efeito significativo no crescimento inicial de plantas, resultando em maior índice de área 
foliar no florescimento e aumento dos componentes do rendimento e produtividade (SCHEERENet al., 2010; TAVARES et al., 2013). Em contra partida, lotes de sementes de qualidade fisiológica reduzida tem como consequência a menor resistência das plântulas no campo a condições adversas (PESKEet al., 2012), podendo contribuir para a redução de produtividade das lavouras.

Uma tecnologia que vem sendo desenvolvida e estudada em nível mundial é a aplicação de aminoácidos em plantas e em sementes com o objetivo de aprimorar adversas ao bom desempenho dos cultivos (LUDWIGet al., 2011; BETTONI et al., 2013; HAMMAD; Ali, 2014; WANG et al., 2014; MONDAL et al., 2015).

Os aminoácidos são ácidos orgânicos cujas moléculas encerram um ou mais grupamento amina, sendo sua principal função constituintes de proteínas, e precursores de várias substâncias que regulam o metabolismo vegetal. A sua aplicação nas diversas culturas não tem o objetivo de suprir a necessidade de aminoácidos para a realização de síntese proteica, mas sim ativar o metabolismo fisiológico das plantas, tendo uma importante ação antiestressante (DA SILVA et al. 2017). Alguns aminoácidos por meiodo seu catabolismo produzem compostos de cadeia carbonada para o ciclo de Krebs, abastecendo assim também o processo de respiração e produção de energia na planta (HILDEBRANDTet al., 2015). Outros são precursores de hormônios vegetais em plantas, como é o caso do triptofano que é precursor do ácido indol acético (AIA) (TAIZ; ZEIGER, 2013) e da metionina precursora do etileno (HILDEBRANDTet al., 2015).

Devido a isso, especula-se que a aplicação de aminoácidos em tratamento de sementes pode promover o desempenho das plantas em campo, principalmente em sementes de baixa qualidade fisiológica. Frente a isso, o objetivo do trabalho foi avaliar o desempenho fisiológico das sementes de soja produzidas por plantas oriundas de sementes de diferentes níveis de qualidade tratadas com doses de aminoácidos.

\section{MATERIAL E MÉTODOS}


Os trabalhos foram desenvolvidos no Laboratório Didático de Análise de Sementes (LDAS) e em área experimental da Faculdade de Agronomia Eliseu Maciel, pertencente à Universidade Federal de Pelotas, situada no município de Capão do Leão, RS.

Os tratamentos envolveram a combinação de dois fatores, sendo eles: fator $A$, lotes de sementes de diferentes níveis de qualidade fisiológica e fator $B$, doses de um produto comercial a base de aminoácidos em tratamento de sementes. Neste sentido, o delineamento experimental adotado foi o de blocos casualizados, em esquema fatorial 3 (fator A) x 5 (fator B), com 4 repetições, totalizando 60 unidades experimentais.

Foram utilizados três lotes de sementes de soja de diferentes níveis de qualidade fisiológica da cultivar BMX Potência RR. O lote de elevada qualidade fisiológica apresentou 94\% de germinação, $82 \%$ de primeira contagem de germinação e 95\% no envelhecimento acelerado; o lote de qualidade fisiológica intermediária apresentou 95\% de germinação, $74 \%$ na primeira contagem de germinação e $76 \%$ no envelhecimento acelerado, enquanto que o lote de baixa qualidade fisiológica apresentou $88 \%$ de germinação, 68\% na primeira contagem de germinação e 57\% no envelhecimento acelerado. Os diferentes níveis de qualidade fisiológica foram obtidos através do envelhecimento artificial das sementes do lote de elevada qualidade fisiológica, permanecendo as sementes 48 e 72 horas para o lote de qualidade fisiológica intermediária e reduzida, respectivamente, sob temperatura de $41^{\circ} \mathrm{C}$ e umidade relativa próxima a $100 \%$.

As sementes dos diferentes níveis de qualidade fisiológica foram tratadas com produto comercial (P.C.) a base de aminoácidos nas doses de 0, 200, 400, 600, e $800 \mathrm{~mL}$ P.C. $100 \mathrm{~kg}^{-1}$ de sementes. O produto utilizado é composto de aminoácidos e nutrientes sendo eles: alanina $(1,164 \%)$, arginina $(0,189 \%)$, ácido aspártico $(1,943 \%)$, ácido glutâmico $(3,316 \%)$, glicina $(0,202 \%)$, isoleucina $(0,171 \%)$, leucina $(0.268 \%)$, lisina $(0,240 \%)$, fenilalanina $(0,143 \%)$, serina $(0,179 \%)$, treonina $(0,188 \%)$, triptofano $(0,175 \%)$, tirosina $(0,122 \%)$, valina $(0,288 \%)$ e os nutrientes: $N-11 \%$ e $\mathrm{K} 2 \mathrm{O}-1 \%$. 
O tratamento de sementes foi realizado colocando o produto, com o auxílio de uma pipeta graduada diretamente no fundo de sacos de polietileno e espalhados pelas laterais dos sacos até altura de $15 \mathrm{~cm}$. Logo após foram adicionadas $0,2 \mathrm{~kg}$ de sementes no interior do saco de polietileno e sendo agitados ambos, produto e sementes, por 3 minutos. Na seqüência, as sementes foram colocadas para secar em temperatura ambiente durante 24 horas.

O experimento foi conduzido em covas espaçadas 30 centímetros, sendo semeadas 10 sementes por cova. Após emergência realizado o desbaste, uniformizando quatro plantas por cova. Previamente à semeadura nas covas, as sementes foram tratadas com inoculante Gelfix na dose de $2 \mathrm{ml} \mathrm{kg}^{-1}$ de sementes. Essas covas foram estabelecidas em canteiros, com dimensões de 6 metros de comprimento e 1 metro de largura, preenchidos com solo coletado do horizonte A1 de um PLANOSSOLO HÁPLICO Eutrófico solódico, (EMBRAPA, 2006) pertencente à unidade de mapeamento Pelotas. A adubação foi realizada de acordo com as recomendações da CFQS RS/SC (Comissão de Fertilidade e Química do Solo RS/SC, 2016), incorporando os nutrientes ao solo cinco dias antes da semeadura.

A avaliação do desempenho fisiológico das sementes produzidas a partir de plantas oriundas de sementes de diferentes níveis de qualidade fisiológica e recobertas com aminoácidos foi realizada por ocasião da maturação nas quatro plantas presentes em cada parcela. As avaliações realizadas foram germinação, primeira contagem de germinação, teste de frio, envelhecimento acelerado, comprimento de raíz e parte aérea de plântulas.

A germinação foi realizada com quatro subamostras de 50 sementes para cada unidade experimental, colocadas em substrato de papel de germinação "germitest", previamente umedecido em água destilada utilizando-se 2,5 vezes a massa do papel seco em peso de água, e mantido à temperatura de $25^{\circ} \mathrm{C}$. As avaliações foram efetuadas conforme as Regras para Análise de Sementes (BRASIL, 2009) e os resultados expressos em porcentagem de plântulas normais. A primeira contagem da germinação constou da determinação da percentagem de plântulas normais aos cinco dias após a semeadura e germinação aos oito dias após a semeadura. 
O teste de frio foi conduzido com quatro subamostras de 50 sementes por unidade experimental, sendo a semeadura realizada em papel-toalha umedecido com água destilada na proporção de 2,5 vezes a massa do papel seco. Os rolos confeccionados foram envoltos por sacos plásticos, lacrados com fita adesiva e mantidos em câmara de germinação (B.O.D) a $10^{\circ} \mathrm{C}$ por sete dias. Ao término desse período, os rolos foram retirados dos sacos plásticos e levados para germinador a 25ํㅡ por cinco dias momento em que foi realizada a avaliação (CÍCERO; VIEIRA, 1994). Os resultados foram expressos em porcentagem de plântulas normais, segundo os critérios adotados para o teste de germinação (BRASIL, 2009).

O envelhecimento acelerado foi realizado utilizando caixa gerbox com tela metálica horizontal fixada na posição mediana. Foram adicionados $40 \mathrm{~mL}$ de água destilada ao fundo de cada caixa gerbox, e sobre a tela foram distribuídas as sementes de cada repetição a fim de cobrir a superfície da tela, constituindo uma única camada. Em seguida, as caixas contendo as sementes foram tampadas e acondicionadas em incubadora do tipo BOD, a $41^{\circ} \mathrm{C}$, onde permaneceram por 48 horas (MARCOS FILHO, 1999). Após este período, as sementes foram submetidas ao teste de germinação, conforme descrito anteriormente.

O comprimento de raiz e parte aérea foi conduzido com quatro subamostras de 20 sementes por unidade experimental, sendo distribuídas em rolos de papel germitest umedecidos com água destilada utilizando-se 2,5 vezes a massa do papel seco, e mantido em germinador a $25^{\circ} \mathrm{C}$, por cinco dias (NAKAGAWA, 1999). Sobre o papel toalha umedecido foi traçado uma linha no terço superior, na direção longitudinal, onde as sementes foram colocadas direcionando-se a micrópila para baixo. O comprimento da raiz primária e das plântulas consideradas normais foi determinado ao final do quinto dia, com o auxílio de régua milimetrada.

Após a coleta e tabulação dos dados, foram verificadas as pressuposições para a análise da variância, e estas sendo atendidas, procedeu-se a análise de variância com o teste $\mathrm{F}$ a $5 \%$ de probabilidade. Para o fator qualitativo (lotes de sementes de diferentes níveis de qualidade fisiológica), quando significativo pelo teste $\mathrm{F}$, as médias foram comparadas pelo teste de Tukey a $5 \%$ de probabilidade. Para o fator quantitativo (doses de aminoácidos) realizou-se análise de regressão, 
sendo testado até o terceiro grau do polinômio. Para o procedimento estatístico utilizou-se o programa Winstat 2.0 (MACHADO; CONCEIÇÃO, 2003).

\section{RESULTADOS}

De acordo com os resultados de desempenho fisiológico das sementes produzidas por plantas oriundas de sementes de diferentes níveis de qualidade fisiológica tratadas com aminoácidos, pode-se observar que não houve interação entre os fatores em estudo (Tabela 1, 2 e 3). Outro aspecto que pode ser observado é que a utilização de aminoácidos não promoveu a produção de sementes de qualidade fisiológica superior. Entretanto, vale salientar, o efeito da qualidade fisiológica das sementes utilizadas na semeadura no desempenho fisiológico das sementes produzidas.

Tabela 1. Germinação e primeira contagem de germinação de sementes de soja produzidas por plantas oriundas de sementes de diferentes níveis de qualidade fisiológica tratadas com doses de aminoácidos, Pelotas - RS, 2018.

Table 1.Germination and first count of germination of soybean seeds produced by plants from different levels of physiological quality treated with doses of amino acids, Pelotas - RS, 2018.

\begin{tabular}{|c|c|c|c|c|c|c|}
\hline \multirow{2}{*}{ Qualidade fisiológica } & \multicolumn{5}{|c|}{ Doses de Aminoácidos ${ }^{1}$} & \multirow{2}{*}{ Média } \\
\hline & 0 & 200 & 400 & 600 & 800 & \\
\hline \multicolumn{7}{|c|}{ Germinação (\%) } \\
\hline Elevada & 98 & 95 & 98 & 98 & 96 & $97 \mathrm{a}$ \\
\hline Intermediária & 97 & 95 & 98 & 95 & 96 & $96 a b$ \\
\hline Reduzida & 94 & 93 & 94 & 93 & 90 & $93 \mathrm{~b}$ \\
\hline Média & $96^{\text {ns }}$ & 94 & 97 & 95 & 94 & \\
\hline C.V. (\%) & & & 3.0 & & & \\
\hline \multicolumn{7}{|c|}{ Primeira Conatgem de Germinação (\%) } \\
\hline Elevada & 96 & 93 & 91 & 92 & 90 & $92 \mathrm{a}$ \\
\hline Intermediária & 96 & 90 & 93 & 94 & 92 & $93 a$ \\
\hline Reduzida & 89 & 87 & 90 & 90 & 85 & $88 \mathrm{~b}$ \\
\hline Média & $94^{\mathrm{ns}}$ & 90 & 91 & 92 & 89 & \\
\hline C.V. (\%) & & & .4 & & & \\
\hline
\end{tabular}

*Médias seguidas da mesma letra minúscula na coluna não diferem pelo teste de Tukey a $5 \%$ de probabilidade. ${ }^{1}$ Doses de aminoácidos em ml P.C. $100 \mathrm{~kg}^{-1}$ de sementes.

ns - não significativo.

C.V. - coeficiente de variação. 
A utilização de sementes de elevada qualidade fisiológica na semeadura promoveu a produção de sementes de soja com maior potencial de germinação e primeira contagem de germinação (Tabela 1.), mas não houve diferença significativa entre sementes de elevada qualidade fisiológica e qualidade intermediária.

Tabela 2. Teste de frio e envelhecimento acelerado de sementes de soja produzidas por plantas oriundas de sementes de diferentes níveis de qualidade fisiológica tratadas com doses de aminoácidos, Pelotas - RS, 2018. Table 2.Cold test and accelerated aging of soybean seeds produced by plants of different levels of physiological quality treated with doses of amino acids, Pelotas - RS, 2018.

\begin{tabular}{|c|c|c|c|c|c|c|}
\hline \multirow{2}{*}{ Qualidade fisiológica } & \multicolumn{5}{|c|}{ Doses de Aminoácidos ${ }^{1}$} & \multirow{2}{*}{ Média } \\
\hline & 0 & 200 & 400 & 600 & 800 & \\
\hline \multicolumn{7}{|c|}{ Teste de Frio (\%) } \\
\hline Elevada & 79 & 80 & 79 & 85 & 88 & $82 \mathrm{a}$ \\
\hline Intermediária & 87 & 82 & 87 & 88 & 79 & $85 \mathrm{a}$ \\
\hline Reduzida & 86 & 88 & 77 & 84 & 85 & $84 \mathrm{a}$ \\
\hline Média & $84^{\mathrm{ns}}$ & 83 & 81 & 86 & 84 & \\
\hline C.V. (\%) & & & 7.4 & & & \\
\hline \multicolumn{7}{|c|}{ רvelhecimento Acelerado (\%) } \\
\hline Elevada & 98 & 93 & 97 & 97 & 95 & $96 \mathrm{a}$ \\
\hline Intermediária & 92 & 91 & 93 & 92 & 94 & $92 \mathrm{~b}$ \\
\hline Reduzida & 93 & 93 & 91 & 88 & 84 & $90 \mathrm{~b}$ \\
\hline Média & $94^{\mathrm{ns}}$ & 92 & 94 & 92 & 91 & \\
\hline C.V. (\%) & & & 4.3 & & & \\
\hline
\end{tabular}

*Médias seguidas da mesma letra minúscula na coluna não diferem pelo teste de Tukey a $5 \%$ de probabilidade. ${ }^{1}$ Doses de aminoácidos em ml P.C. $100 \mathrm{~kg}^{-1}$ de sementes.

ns - não significativo.

C.V. - coeficiente de variação.

Com relação aos resultados dos testes de vigor das sementes produzidas constata-se também efeito da qualidade fisiológica das sementes utilizadas na semeadura no vigor das sementes produzidas. Plantas oriundas de sementes de elevada qualidade fisiológica produziram sementes que apresentaram desempenho no teste de frio, envelhecimento acelerado e comprimento de plântulas e suas partes superior (Tabelas 2, 3).Sementes de elevada qualidade fisiológica não diferiram das demais quando submetidas ao teste de frio. 
Tabela 3. Comprimento de parte aérea e radicular de sementes de soja produzidas por plantas oriundas de sementes de diferentes níveis de qualidade fisiológica tratadas com doses de aminoácidos, Pelotas - RS, 2018. Table 3.Aerial and root length of soybean seeds produced by plants from different levels of physiological quality seed treated with doses of amino acids, Pelotas - RS, 2018.

\begin{tabular}{|c|c|c|c|c|c|c|}
\hline \multirow{2}{*}{ Qualidade fisiológica } & \multicolumn{5}{|c|}{ Doses de Aminoácidos $^{1}$} & \multirow{2}{*}{ Média } \\
\hline & 0 & 200 & 400 & 600 & 800 & \\
\hline \multicolumn{7}{|c|}{ Comprimento de Parte Aérea (mm) } \\
\hline Elevada & 99 & 91 & 99 & 94 & 96 & $96 \mathrm{a}$ \\
\hline Intermediária & 90 & 89 & 93 & 89 & 82 & $89 \mathrm{~b}$ \\
\hline Reduzida & 91 & 89 & 86 & 85 & 92 & $89 \mathrm{~b}$ \\
\hline Média & $93^{\text {ns }}$ & 90 & 93 & 89 & 90 & \\
\hline C.V. (\%) & & & & & & \\
\hline \multicolumn{7}{|c|}{ Comprimento Radicular (mm) } \\
\hline Elevada & 143 & 127 & 134 & 134 & 135 & $135 \mathrm{a}$ \\
\hline Intermediária & 132 & 121 & 134 & 130 & 125 & $128 a b$ \\
\hline Reduzida & 129 & 127 & 121 & 117 & 123 & $123 b$ \\
\hline Média & $135^{\mathrm{ns}}$ & 125 & 130 & 127 & 128 & \\
\hline C.V. (\%) & \multicolumn{5}{|c|}{7.9} & \\
\hline
\end{tabular}

\section{DISCUSSÃO}

Os resultados obtidos na tabela 1 discordam dos encontrados na literatura até o momento, que não identificam efeito da qualidade fisiológica das sementes utilizadas na semeadura sobre a germinação e primeira contagem de germinação das sementes produzidas (TAVARESet al., 2013; ROSSI et al., 2017).

Nas tabelas 2 e 3 os resultados demonstram que 0 efeito da qualidade fisiológica das sementes utilizadas na semeadura transmitiu-se as sementes produzidas pelas plantas delas originadas, resultando que o uso de sementes de elevada qualidade fisiológica proporcionou ao final do ciclo de cultivo a obtenção de sementes de qualidade fisiológica superior.

O efeito da qualidade fisiológica de sementes no crescimento inicial de plantas, na uniformidade da lavoura, desempenho de plantas e inclusive na 
produtividade de grãos tem sido bastante estudado e comprovado (KOLCHINSKI et al., 2006; SCHEEREN et al., 2010; TAVARES et al., 2013; CANTARELI et al., 2015). Entretanto, efeitos sobre a transmissão da influência da qualidade fisiológica sobre as sementes produzidas e/ou sobre o comportamento da geração seguinte são mais escassos (TAVARESet al., 2013; ROSSI et al., 2017).

Tavares et al. (2013) não identificaram diferenças na qualidade fisiológica das sementes produzidas devido ao efeito de qualidade das sementes utilizadas na semeadura e atribuiu a este resultado o fato de que as plantas oriundas de sementes de baixo vigor produzem menor área foliar mas também menor número de sementes por planta e assim a quantidade de fotoassimilados disponível por semente foi a mesma que de plantas oriundas de sementes de elevado vigor que apresentam um maior aparato fotossintético e maior número de sementes por planta. Rossi et al. (2017) também não encontraram efeito da qualidade fisiológica das sementes utilizadas na semeadura no desempenho fisiológico das sementes produzidas.

$\mathrm{Na}$ produção de sementes de soja são diversos os fatores que podem influenciar o crescimento e desenvolvimento das plantas, e essas modificações podem refletir na qualidade fisiológica das sementes produzidas. Diversos trabalhos evidenciam a influência de diferentes fatores na qualidade fisiológica das sementes produzidas, tais como condições do solo, nutrição de plantas, incidência de doenças, condições do ambiente como períodos de seca, excesso de chuvas, extremos de temperatura e fortes flutuações das condições de umidade ambiente na maturação, facilitando o aparecimento de sementes com altos índices de deterioração (FRANÇA NETO et al., 2007; PESKE et al., 2012). Porém poucos trabalhos têm evidenciado a influência da qualidade fisiológica das sementes utilizadas na semeadura sobre a qualidade fisiológica das sementes produzidas. A qualidade fisiológica das sementes utilizadas no processo de semeadura influencia todo crescimento e desenvolvimento inicial da cultura e estas diferenças persistem até o fim da estação de crescimento da cultura (KOLCHINSKI et al., 2006; SCHEEREN et al., 2010; TAVARES et al., 2013). 
Plantas oriundas de sementes de elevada qualidade fisiológica emergem antecipadamente, adquirem capacidade de utilizar os recursos do ambiente antecipadamente, além de propiciar um maior período vegetativo das culturas, promovendo maior acúmulo de fotoassimilados pelo maior influxo de $\mathrm{CO}_{2}$ (FLOSS, 2008). Possivelmente, as plantas oriundas de sementes de elevada qualidade fisiológica encontraram-se no estádio reprodutivo com maior aporte fotossintético disponível para a formação das sementes.

\section{CONCLUSÕES}

Plantas de soja oriundas de sementes de elevada qualidade fisiológica produzem sementes de desempenho fisiológico superior.

O tratamento das sementes de soja utilizadas na semeadura com doses de aminoácidos não apresenta efeito significativo no desempenho fisiológico das sementes produzidas.

\section{REFERÊNCIAS}

BETTONI, M.M.; FABBRIN, E.G.S.; OLINIK, J.R.; MÓGOR, Á. F. Efeito da aplicação foliar de hidrolisado proteico sob a produtividade de cultivares de brócolis. RevistaAgro@mbiente On-line, v.7, n.2, p.179-183, 2013. DOI: http://dx.doi.org/10.18227/1982-8470ragro.v7i2.943.

BRASIL. Ministério da Agricultura, Pecuária e Abastecimento. Regras para análise de sementes. Brasília: Mapa/ACS, 2009.395 p.

CANTARELLI, L. D.; SCHUCH, L. O. B.; RUFINO, C. A.; TAVARES, L. C.; VIEIRA, J. F. Physiological seeds quality: spatial distribution and variability among soybean plant population. Bioscience Journal, v.31, n.2, p.344-351, 2015. DOI: http://dx.doi.org/10.14393/BJ-v31n2a2015-22274

CÍCERO, S.M.; VIEIRA, R.D. Teste de frio. In: VIEIRA, R.D.; CARVALHO, N.M. Testes de vigor em sementes. Jaboticabal: FUNEP, 1994. p.151-164.

COMISSÃO DE QUÍMICA E FERTILIDADE DO SOLO - RS/SC. Manual de Adubação e de Calagem para os estados do Rio Grande do Sul e de Santa Catarina. $11^{\circ}$ ed. Porto Alegre: NRS/SBCS, 2016. 400p. 
COMPANHIA NACIONAL DE ABASTECIMENTO. Acompanhamento de safra brasileira: Grãos, 12ª Levantamento. Setembro/2017. Brasília: CONAB, 134 pp.

DA SILVA, N. F.; CLEMENTE, G. S.; TEIXEIRA, M. B.; SOARES, F. A. L.; CUNHA, F. N.; AZEVEDO, L. O. da S. Use of foliar fertilizers for the specific physiological management of different soybean crop stages. American Journal of Plant Sciences.v.8, n.4, p.810-834, 2017.DOI: https://doi.org/10.4236/ajps.2017.84056

EMPRESA BRASILEIRA DE PESQUISA AGROPECUÁRIA - EMBRAPA. Centro Nacional de Pesquisa de Solos. Sistema brasileiro de classificação de solos. $2^{\circ}$ ed. Rio de Janeiro, 2006. 306p.

FLOSS, E.L. Fisiologia das plantas cultivadas. ed. 4. Passo Fundo-RS: UPF, 2008.749p.

FRANÇA NETO, J.; KRZYZANOWSKI, F.; PÁDUA, G.; COSTA, N.; HENNING, A. Tecnologia da produção de semente de soja de alta qualidade: Série Sementes.Londrina: EmbrapaSoja. 2007. 12p. (Circular Técnica 40).

HAMMAD, S.A. R.; ALI, O.A.M. Physiological and biochemical studies on drought tolerance of wheat plants by application of amino acids and yeast extract. Annals of Agricultural Science.v.59, n.1, p.133-145, 2014.DOI: https://doi.org/10.1016/j.aoas.2014.06.018

HILDEBRANDT, T. M.; NESI, A. N.; ARAÚJO, W. L.; BRAUN, H. P. Amino acid catabolism in plants. Molecular Plant.v.8, p.1563-1579, 2015. DOI: https://doi.org/10.1016/j.molp.2015.09.005

KOLCHINSKI, E.M.; SCHUCH, L.O.B; PESKE, S.T.Crescimento inicial de soja em função do vigor das sementes. Revista Brasileira de Agrociência.v.12, n.2, p. 163166, $2006 . \quad$ Disponível em $<$ http://www2.ufpel.edu.br/faem/agrociencia/v12n2/artigo07.pdf >. Acesso: 31 de julho de 2018.

LUDWIG, M.P.; FILHO, O.A.L.; BAUDET, L.; DUTRA, L.M.C.; AVELAR, S.A.G.; CRIZEL, R.L.Qualidade de sementes de soja armazenadas após recobrimento com aminoácido, polímero,fungicida e inseticida. Revista Brasileira de Sementes.v.33, n.3, p. 395-406, 2011. Disponível em :<http://www.scielo.br/pdf/rbs/v33n3/02.pdf $>$. Acesso: 28 de julho de 2018.

MACHADO, A.A.; CONCEIÇÃO, A.R. Sistema de análise estatística para windows. WinStat. Versão 2.0. Pelotas: UFPel, 2003.

MARCOS FILHO, J. Teste de envelhecimento acelerado. In: KRZYZANOWSKI, F.C.; VIEIRA, R.D.; FRANÇA NETO, J.B. (Ed.) Vigor de sementes: conceitos e testes.Londrina: ABRATES, 1-24, 1999. 
MONDAL, M.F.; ASADUZZAMAN, M.; TANAKA, H., ASAO, T. Effects of amino acids on the growth and flowering of Eustoma grandiflorum under autotoxicity in closed hydroponic culture.ScientiaHorticulturae. v.192, p. 453-459, 2015.DOI: https://doi.org/10.1016/j.scienta.2015.05.024

NAKAGAWA, J. Testes de vigor baseados na avaliação de plântulas. In: KRZYZANOWSKI, F.C.; VIEIRA, R.D.; FRANÇA-NETO, J.B. (Ed.). Vigor de sementes: conceitos e testes. Londrina: ABRATES, 1-21, 1999.

PESKE, S.T.; VILLELA, F. A.; MENEGHELLO, G. E. Sementes: Fundamentos Científicos e Tecnológicos. $2^{\circ}$ ed. Pelotas, UFPel. 2012.573 p.

ROSSI, R. F.; CAVARIANI, C.; FRANÇA-NETO, J. D. Vigor de sementes, população de plantas e desempenho agronômico da soja. Amazonian Journal of Agricultural and Environmental Sciences.v.60, n.3, p.215-222, 2017.DOI: http://dx.doi.org/10.4322/rca.2239

SCHEEREN, B.R.; PESKE, S.T.; SCHUCH, L.O.B.; BARROS, A.C.S.A. Qualidade fisiológica e produtividade de sementes de soja. Revista Brasileira de Sementes. v.32, n.3, p.035-041, 2010. Disponível em: $<$ http://www.scielo.br/pdf/rbs/v32n3/v32n3a04.pdf >. Acesso: 22 de julho de 2018.

TAIZ, L. \& ZEIGER, E. Fisiologia Vegetal. 5를 edição. 2013.782 p.

TAVARES, L.C.; RUFINO, C.A.; BRUNES, A.P.; TUNES, L.M.; BARROS, A.C.S.A.; PESKE, S.T. Desempenho de sementes de soja sob deficiência hídrica: rendimento e qualidade fisiológica da geração F1. Ciência Rural.v.43, n.8, p.1357-1363, 2013. Disponível em: <http://www.scielo.br/pdf/cr/v43n8/a22213cr2012-0950.pdf >. Acesso: 18 de junho de 2018.

WANG, J.; LIU, Z.; WANG, Y.; CHENG, W.; MOU, H. Production of a water soluble fertilizer containing amino acids by solid-state fermentation of soybean meal and evaluation of its efficacy on the rapeseed growth. Journal of Biotechnology, v.187, p.34-42, 2014. DOI: https://doi.org/10.1016/j.jbiotec.2014.07.015 